\title{
Hallmark trials in ANCA-associated vasculitis (AAV) for the pediatric rheumatologist
}

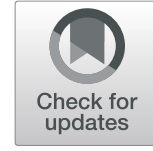

Jennifer J. Y. Lee ${ }^{1 \dagger}$, Alhanouf Alsaleem ${ }^{1 \dagger}$, Grace P. K. Chiang ${ }^{1,2 \dagger}$, Elizaveta Limenis ${ }^{1 \dagger}$, Watchareewan Sontichai ${ }^{1,3 \dagger}$, Rae S. M. Yeung ${ }^{1}$, Jonathan Akikusa ${ }^{4}$ and Ronald M. Laxer ${ }^{1 *}$

\begin{abstract}
Anti-neutrophil cytoplasmic antibody (ANCA)-associated vasculitis (AAV) refers to a complex group of systemic vasculitides that are characterized by primary small-to-medium sized blood vessel inflammation with the presence of autoantibodies known as ANCA. AAV diseases include Granulomatosis with Polyangiitis (GPA), Eosinophilic Granulomatosis with Polyangiitis (EGPA), and Microscopic Polyangiitis (MPA). AAVs are challenging conditions associated with high cumulative disease and treatment related morbidity and mortality. Given its rarity and the resulting paucity of pediatric-specific clinical trial evidence, pediatric rheumatologists have had to often extrapolate from adult literature for management and therapeutic decisions. The aim of this review is to provide a comprehensive overview of the important findings and overall conclusions of critical landmark clinical trials in the induction and maintenance treatments in adult AAV for the pediatric rheumatologist. This review also highlights the outcomes of recent pediatric AAV observational studies and discusses the future research priorities in pediatric AAV management.
\end{abstract}

Keywords: Pediatric, Vasculitis, Anti-neutrophil cytoplasmic antibody-associated Vasculitis, Management

\section{Background}

Systemic vasculitis is a challenging and complex multiorgan disease that results in primary inflammation of the blood vessel wall. Anti-neutrophil cytoplasmic antibody (ANCA)-associated vasculitis (AAV) is a group of systemic vasculitides that is characterized by small-to-medium sized blood vessel inflammation with the presence of autoantibodies known as ANCA. AAV diseases include Granulomatosis with Polyangiitis (GPA), Eosinophilic Granulomatosis with Polyangiitis (EGPA), and Microscopic Polyangiitis (MPA). AAVs are one of the more common types of systemic vasculitis encountered by pediatric rheumatologists. Proper treatment of this condition is critical as the mortality of untreated AAV can be up to $80 \%$ [1,2]. Given the paucity of clinical trials in pediatric AAV, pediatric rheumatologists have relied on adult AAV evidence for management. In this

\footnotetext{
* Correspondence: ronald.laxer@sickkids.ca

Jennifer J. Y. Lee, Alhanouf Alsaleem, Grace P. K. Chiang, Elizaveta Limenis, and Watchareewan Sontichai are first authors.

${ }^{1}$ Department of Pediatrics, Division of Rheumatology, Hospital for Sick

Children and University of Toronto, 555 University Avenue, Toronto, ON M5G

$1 \times 8$, Canada

Full list of author information is available at the end of the article
}

review, we highlight key findings of critical landmark trials in AAV for the pediatric rheumatologist.

\section{Disease activity assessments}

Standardized tools are important in measuring disease activity and damage; they also help guide treatment decisions in rheumatic diseases. Numerous instruments have been developed to measure disease activity in AAV [3-5]. These measurements are often used to define primary or secondary outcomes in AAV trials. Thus, it is important for pediatric rheumatologists to familiarize themselves with these tools.

\section{Birmingham Vasculitis activity score}

The Birmingham Vasculitis Activity Score (BVAS), originally published in 1994 and then revised in 1997 and 2009, is the most widely used tool in clinical practice and trials $[6$, 7]. The BVAS is a composite score that evaluates 56 clinical features from 9 organ systems that are attributed to active vasculitis. Each item is weighted according to the severity. A score of 0 is often adopted as the definition of disease remission in studies. The revised BVAS acknowledges persistent symptoms in addition to new and worsening 
symptoms. A variation of the BVAS available for GPA patients is known as the BVAS/WG [8]. This score has greater disease specificity in patients with GPA but cannot be generalized to other types of systemic vasculitis.

\section{Pediatric Vasculitis activity score}

There had been no validated tool for assessment of disease activity in pediatric patients with systemic vasculitis until 2012 [9], when international collaborative efforts led to the development and validation of a pediatric vasculitis assessment tool. The Pediatric Vasculitis Activity Score (PVAS) is modified from the BVAS. In the PVAS, 22 original BVAS items were redefined and 8 new items were added, resulting in 64 clinical items grouped under 9 organ systems. Every item has an assigned score in the 'new/worse' and 'persistent' scale. This score was used in a recent study to measure the early outcomes in children with AAV [10].

\section{Disease damage assessments}

Measurement of damage is an essential component in the follow-up assessments of chronic disease. The Vasculitis Damage Index (VDI) [11] is an unweighted scoring system comprising 64 items grouped under 11 organ-based systems. Damage is defined as an irreversible change lasting for more than 3 months. The VDI is a cumulative index and can only remain static or increase over time. The damage recorded needs to occur after the vasculitis diagnosis, but do not need to be attributable to the diagnosis (e.g. might be related to treatment).

There is no validated tool to assess disease damage in children with vasculitis. However, the Pediatric Rheumatology European Society (PRES) Vasculitis Working Group and Childhood Arthritis \& Rheumatology Research Alliance (CARRA) are working toward validating a formal pediatric modification of the VDI [10] - PVDI, which has been piloted in some studies.

\section{Treatment overview}

Treatment of AAV is generally categorized into two phases: induction and maintenance. Induction therapy refers to the therapies required to achieve disease remission. After achieving remission, maintenance therapy is initiated to prevent relapses. This review discusses induction therapy for severe and limited AAV separately, followed by maintenance therapy trials.

The European League Against Rheumatism (EULAR) has previously described more specific disease severity definitions by expert consensus in order to conduct trials with more homogeneous AAV patient populations, and to make trials comparable (Table 1) [12]. For clinically relevant purposes, severe (also described as generalized) AAV is defined as presence of life- or major organ-threatening manifestations. The Canadian Vasculitis Research Network (Canvasc) recommendations on AAV management provides clinical examples describing major organ-threatening manifestations, which may include severe and progressive kidney involvement, alveolar haemorrhage resulting in severe hemoptysis, severe gastrointestinal (e.g. intestinal bleeding), cardiac (e.g. heart failure due to pericarditis or myocarditis), central nervous system (e.g. rapidly progressive neuropathy), or ocular involvement (e.g. orbital pseudotumor) [13]. Limited AAV is often defined as localized involvement without organ-threatening manifestations. Patients with constitutional symptoms are generally included. Patients with renal or pulmonary manifestations may be included with the caveat that the organ manifestations do not result in threatened organ function (e.g. pulmonary infiltrates without severe hemoptysis or mildly reduced kidney function with a $\mathrm{Cr}<120$ without evidence of casts or significant proteinuria).

\section{Induction trials}

Induction trials for severe disease

Several trials have addressed induction regimens for severe AAV (Table 2).

Table 1 Disease Severity Definitions

\begin{tabular}{|c|c|c|c|c|c|}
\hline Study Group & $\begin{array}{l}\text { Clinical } \\
\text { Subgroup }\end{array}$ & $\begin{array}{l}\text { Systemic Vasculitis Outside Ears, } \\
\text { Nose, Throat and Lungs }\end{array}$ & $\begin{array}{l}\text { Threatened Vital } \\
\text { Organ Function }\end{array}$ & Other Definitions & Serum Creatinine (umol/L) \\
\hline \multirow[t]{5}{*}{ EUVAS } & Localized & No & No & $\begin{array}{l}\text { No constitutional symptoms, } \\
\text { ANCA typically negative }\end{array}$ & $<120$ \\
\hline & $\begin{array}{l}\text { Early } \\
\text { Systemic }\end{array}$ & Yes & No & $\begin{array}{l}\text { Constitutional symptoms present, } \\
\text { ANCA-positive or negative }\end{array}$ & $<120$ \\
\hline & Generalized & Yes & Yes & ANCA-positive & $<500$ \\
\hline & Severe & Yes & Organ Failure & ANCA-positive & $>500$ \\
\hline & Refractory & Yes & Yes & Refractory to standard therapy & Any \\
\hline \multirow[t]{2}{*}{$\begin{array}{l}\text { WGET Research } \\
\text { Group }\end{array}$} & Limited & Allowed, but not required & No & Not severe & $\begin{array}{l}<124 \text {, if hematuria, but no red } \\
\text { blood cell casts present }\end{array}$ \\
\hline & Severe & Yes & Yes & Organ- or life-threatening disease & Any \\
\hline
\end{tabular}

From Hellmich et al., EULAR Recommendations for conducting clinical studies and/or clinical trials in systemic vasculitis: focus on anti-neutrophil cytoplasm antibody-associated vasculitis. [12] 


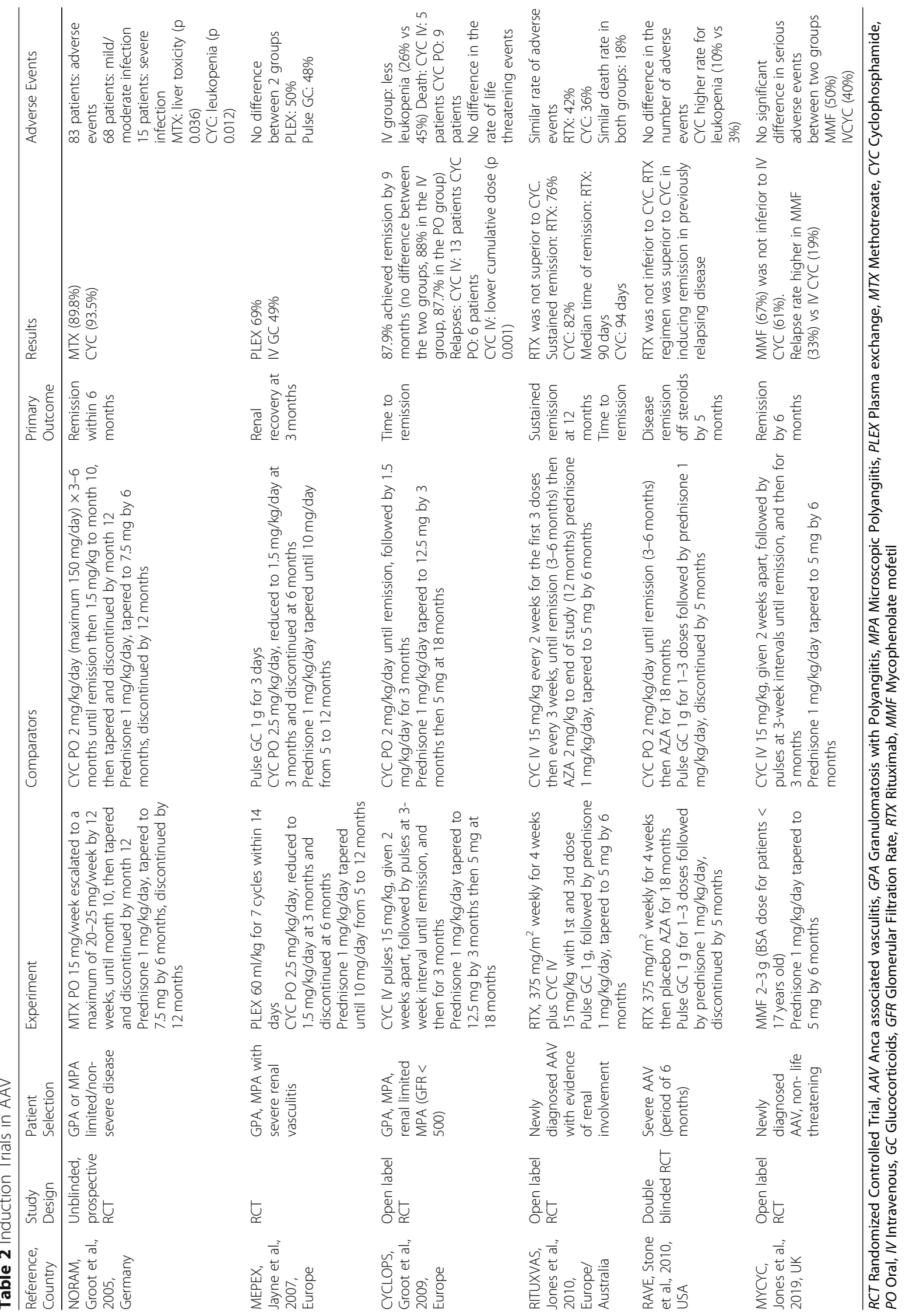


Cyclophosphamide (CYC) is one of the most commonly used and well-studied induction therapies. All CYC trials were conducted with one shared aim: to achieve disease remission and minimize medication toxicity. CYCLOPS was an open label multicenter randomized controlled trial (RCT) that evaluated the effect of intermittent intravenous (ivCYC) versus daily oral CYC (poCYC) [14]. The primary outcome was time to remission, defined as the absence of new or worse signs of disease activity on the BVAS and no more than 1 item indicating persistent disease activity (BVAS $\leq 1)$. The time to remission did not differ between groups (median 3 months). The authors concluded that the use of ivCYC in comparison to poCYC had similar efficacy in achieving remission, but ivCYC therapy had the advantage of having a lower toxicity profile with a reduced cumulative dose. However, a long-term follow-up study with a median duration of 4.3 years showed higher relapse rates with ivCYC compared to poCYC. Interestingly, antiproteinase-3 (PR3) positive patients had higher relapse rates in both groups.

Rituximab (RTX), an anti-CD20 monoclonal antibody and B-cell depleting agent, has emerged as a new therapeutic agent for AAV. RITUXVAS is a multicenter RCT in which newly diagnosed AAV patients with renal disease were randomized to receive ivCYC for 3-6 months, or RTX weekly for 4 weeks plus ivCYC with the first and third RTX infusion. All patients received $1 \mathrm{~g}$ of methylprednisolone followed by a tapering course of oral corticosteroids, with reduction to $5 \mathrm{mg}$ by 6 months [15]. The primary outcome was rates of sustained remission (BVAS of 0 for at least 6 months) and rates of serious adverse events (AEs) at 12 months. The RTX-based induction regimen was not superior to ivCYC. The longterm follow-up study also reported no difference in disease remission, AEs, or mortality [16].

The RAVE (Rituximab in ANCA-Associated Vasculitis) trial evaluated RTX in comparison to poCYC utilizing a non-inferiority trial design [17]. This trial differed from the RITUXVAS trial in that the participants of the RAVE trial were younger with less severe renal disease. Furthermore, the comparator agent was oral as opposed to intravenous CYC. The ambitious primary outcome was to achieve disease remission (BVAS of 0 ) off corticosteroids by 6 months. Both groups had similar corticosteroid regimens. Sixty-four percent of patients in the RTX group had disease remission in comparison to $53 \%$ in the poCYC group. This result met the definition for non-inferiority. However, remission rates were overall lower in comparison to other studies, possibly attributable to the earlier discontinuation of corticosteroids. In subsequent analyses, subgroups of patients with either relapsing disease at baseline or those with anti-PR3 positivity achieved higher response rates with RTX when compared to CYC. A long-term follow-up of the RAVE cohort showed that in patients with severe AAV without organ failure, RTX regimen was equivalent to CYC in maintaining disease remission after 18 months [18].

Plasma exchange (PLEX) is a non-pharmacologic treatment considered in patients with severe AAV. The MEPEX trial compared the addition of either IV corticosteroids or PLEX in patients with AAV who had severe renal vasculitis (serum creatinine $>500 \mu \mathrm{mol} / \mathrm{L}$ or dialysis dependence). The primary outcome was renal recovery (dialysis independence) at 3 months, which was achieved in 49 and $69 \%$ in the corticosteroid and PLEX arm, respectively [19]. PLEX decreased the incidence of end stage renal disease (ESRD) from 43 to $24 \%$ at 12 months, but this difference was lost after long-term follow-up [20]. PEXIVAS is an international, open-label, two-by-two factorial design study that recruited patients with new or relapsing severe AAV to investigate the use of adjunctive PLEX with standard therapy (CYC or RTX) and two different corticosteroid regimens (standard or low dose) [19]. The primary outcome was a composite measure of death from any cause or ESRD. Preliminary results from 704 patients found that the primary outcome occurred in $28 \%$ in the PLEX arm compared to $31 \%$ in the no-PLEX arm. [21]. Interestingly, varying the steroid regimens did not result in a difference to the primary outcome.

\section{Induction trials for limited disease}

There has been interest in identifying safer immunosuppressive regimens in the management of patients with limited AAV. The NORAM (Non-Renal vasculitis Alternatively treated with Methotrexate), a non-blinded RCT, hypothesized that induction with oral Methotrexate (poMTX) can spare the toxicity of poCYC in early systemic AAV [22]. The primary outcome was disease remission by 6 months, with tapering of induction therapy by 12 months. All patients were treated with the same corticosteroid regimen. The NORAM trial demonstrated that poMTX was not inferior to poCYC. However, at 18 months, relapse rates were significantly higher in the poMTX group, suggesting that when given as an alternative to poCYC, poMTX may need to be given for longer than 12 months. Long-term follow-up of the cohort (median 6 years) found no difference with respect to AEs between the two regimens and higher relapse rates in the poMTX-treated group [23].

Mycophenolate mofetil (MMF) has been studied for induction therapy for limited disease. MYCYC (MMF versus $\mathrm{CYC}$ for remission induction of $\mathrm{AAV}$ ) is a randomized non-inferiority trial that compared MMF with ivCYC. The primary outcome was the proportion of patients achieving remission (BVAS of 0 ) by 6 months. The MMF group received doses ranging from 2 to $3 \mathrm{~g}$ and 
the ivCYC group was treated with a similar regimen used in the CYCLOPS trial. Both groups received the same corticosteroid regimen. Sixty-seven percent of patients in the MMF group achieved the primary outcome in comparison to $61 \%$ in the ivCYC group. Following remission, relapses occurred significantly more frequently with MMF (33\%) compared to ivCYC (19\%). The authors concluded that MMF was non-inferior to ivCYC, but that MMF may result in more relapses [24].

\section{Maintenance trials}

Given the concerns of utilizing CYC long-term, such as increased risk of malignancy and infertility, investigators have evaluated the use of less toxic immunosuppressants as alternatives (Table 3). In the CYCAZAREM (CYC versus AZA for Early REMission phase of vasculitis) trial, patients with a newly diagnosed severe AAV and a serum creatinine of $\leq 500 \mu \mathrm{mol} / \mathrm{L}$ in whom remission had been achieved within 3-6 months, were randomly assigned to continue poCYC or switch to azathioprine (AZA) for 12 months. All subjects were then switched to a lower dose of AZA, which continued to the end of the study (18 months). Both groups continued a tapering course of corticosteroids. The primary outcome was major and minor relapse rate at 18 months. Relapse rates were not significantly different between groups [25]. There was no difference in severe AEs, although the study was not powered to detect differences in $\mathrm{AE}$ rates. Long-term follow-up (median 8.5 years) revealed a trend for worse outcomes in the AZA group in terms of relapses and development of ESRD but these were not statistically significant [26].

While the CYCAZAREM study validated AZA as a suitable alternative to poCYC for maintenance, the optimal duration of AZA treatment was not examined. The REMAIN (prolonged REmission-MAINtenance therapy in systemic vasculitis) trial concluded that prolonged maintenance therapy with AZA and low dose corticosteroids to 48 months from diagnosis resulted in a 3-fold reduction in the frequency of relapses compared with withdrawal of AZA and corticosteroids by 24 months [27]. Moreover, the continuation group had improved renal survival with reduced incidence of ESRD. In a RCT (AZA-ANCA trial) comparing standard and extended AZA maintenance therapy in patients with PR3-AAV, patients treated with longer treatment duration (4 years after diagnosis and tapered thereafter) had a lower relapse rate, albeit not significant, compared with those treated with standard treatment (1 year after diagnosis and tapered thereafter) [28]. However, this trial was terminated prematurely given slow patient recruitment and did not achieve an adequate sample size. Therefore, although not definitively proven, a longer duration of maintenance therapy may lead to better outcomes.

The use of MTX as an alternative maintenance agent with possibly less toxicity and equal or perhaps greater efficacy than AZA was examined in the WEGENT trial. In the WEGENT trial, AAV patients in remission were randomized to either AZA or MTX and a tapering oral steroid course [29]. The rate of AEs causing death or study withdrawal was the same between the two groups, indicating MTX was similar in toxicity with AZA. Relapse rates were also similar confirming MTX as a viable alternative to AZA. In the follow-up study, 10-year overall survival rates, total number of relapses, relapse rates and AEs did not differ significantly [30].

Other immunosuppressive agents, including MMF and leflunomide, have also been studied. The IMPROVE (International MMF Protocol to Reduce Outbreaks of Vasculitides) trial compared the efficacy of MMF versus AZA for maintenance of remission in AAV patients in whom remission had been induced with corticosteroids and CYC, with or without methylprednisolone pulses and PLEX. MMF was significantly less effective at preventing relapses when compared to AZA after a median follow-up of 39 months [31]. In a RCT comparing leflunomide and MTX, leflunomide was found to be more effective than MTX in preventing major relapses but was associated with more AEs [32].

The MAINRITSAN (Maintenance of Remission using Rituximab in Systemic ANCA-Associated Vasculitis) trial is the first RCT to evaluate RTX for maintenance therapy. After achieving remission, patients were randomly assigned to receive either RTX at 0 and 2 weeks following randomization then every 6 months until 18 months or AZA until 22 months. Both groups were treated with corticosteroids for at least 18 months. There were significantly fewer major relapses at 28 months in the RTX group compared with the AZA group (5\% versus 29\%) [33]. In the long-term study, RTX remained superior to AZA up to 60 months, with greater rates of relapse-free and overall survival [34]. The MAINRITSAN2 compared an individually tailored RTX regimen with fixed-schedule regimen [35]. Patients in the tailored-infusion arm received RTX at randomization and received repeat infusions based on lymphocyte counts and ANCA titers until 18 months. The fix-scheduled arm received the same regimen from the original MAINRITSAN trial. There was no significant difference between the number of relapses but the tailoredinfusion arm received fewer number of infusions overall.

The BREVAS (Belimumab in Remission of Vasculitis) is a recent $\mathrm{RCT}$ that evaluated the efficacy of belimumab, a monoclonal antibody against B lymphocyte stimulator, as an adjunctive therapy to a regimen of AZA with low-dose corticosteroids [36]. Overall, the addition of belimumab did not reduce relapses.

\section{Eosinophilic granulomatosis with Polyangiitis (EGPA)}

EGPA treatment recommendations are less robust due to the lack of RCTs. The treatment is often inferred 


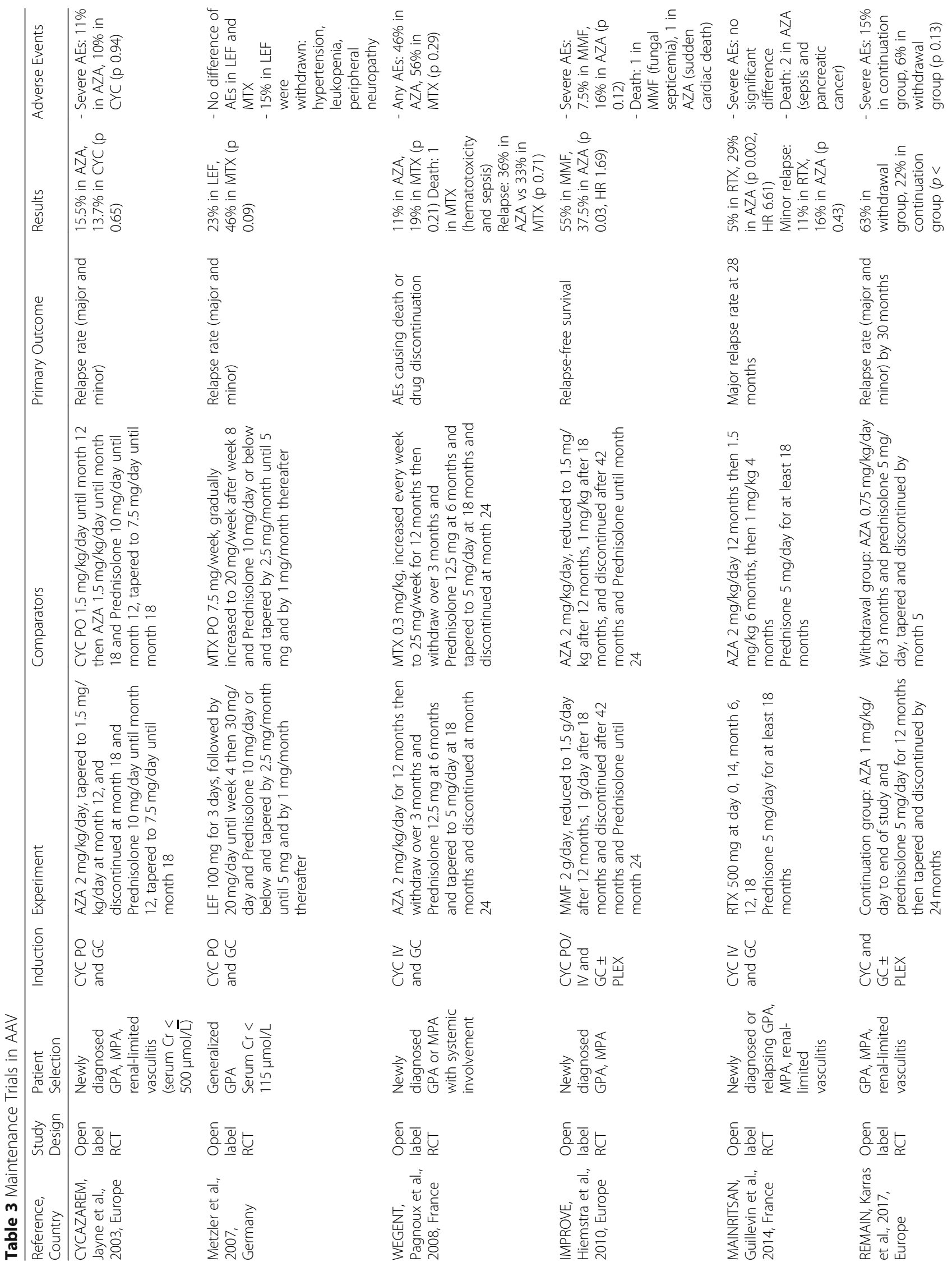


$\begin{array}{ll}\text { Lee et al. Pediatric Rheumatology } & \text { (2019) 17:31 }\end{array}$

Page 7 of 15

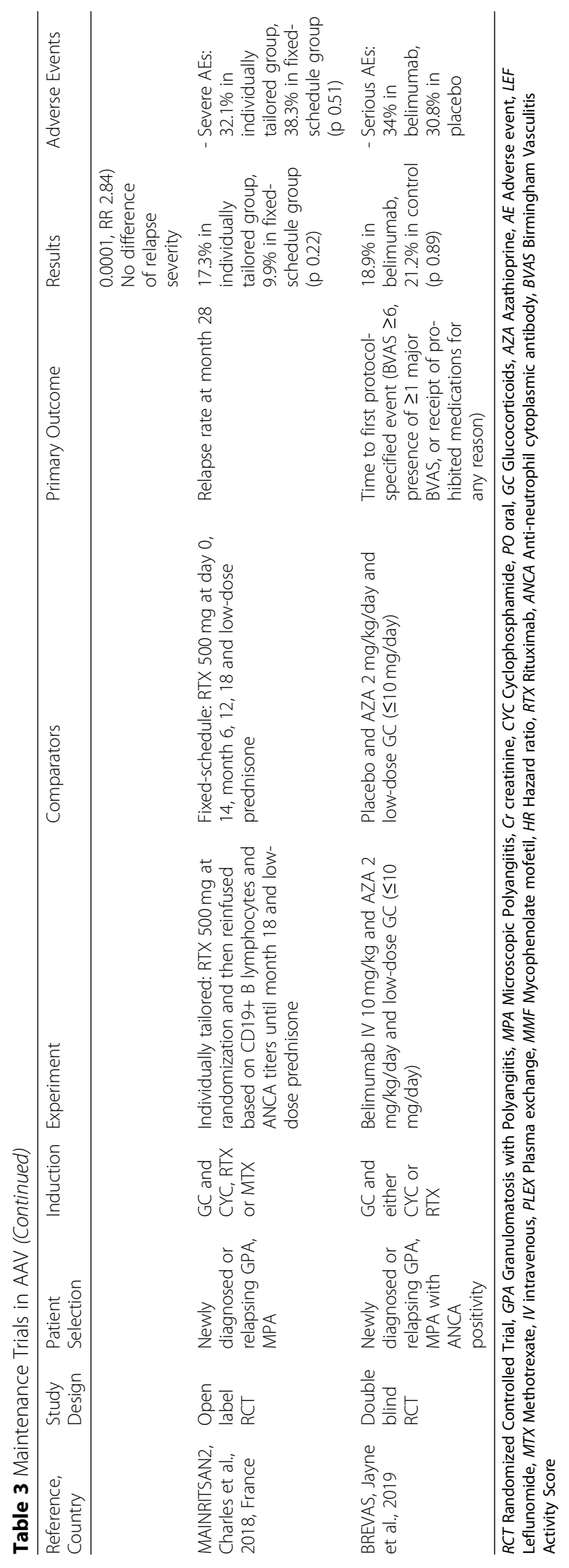


from GPA/MPA trials, although EGPA patients were either excluded or present in small numbers in these studies.

Only a few trials have studied immunosuppressive therapy in larger numbers of EGPA patients (Table 4) [37-40]. The most commonly studied agent for EGPA has been CYC, although studies have largely looked at variations in CYC regimens as opposed to comparing the efficacy of CYC to other immunosuppressants. Currently, the EGPA Consensus task force recommends the use of CYC for patients with organ threatening disease for induction [41].

Based on a prospective cohort study, it is recommended that EGPA patients without life or organ threatening manifestations can be treated with corticosteroid monotherapy because of excellent 5-year survival rates, although relapse rates are high on a monotherapy regimen [42]. Puechal et al. performed a trial that evaluated the addition of AZA to corticosteroids for induction [43]. The study concluded no additional accrued benefits by adding AZA, with no differences in induction failures or relapses within 2 years ( $47 \%$ versus $49 \%$ ) [41]. It is likely that a different adjunctive therapy is required for induction in non-severe EGPA patients.

Unique to EGPA is the potential role of interleukin-5 (IL-5) blockade. Wechsler et al. performed a RCT on the use of mepolizumab, an anti-IL-5 monoclonal antibody, in patients with relapsing or refractory EGPA [44]. Participants were either randomized to mepolizumab or to placebo. The two primary end points were the accrued weeks of remission at 52-weeks and the proportion of patients in remission at weeks 36 and 48. The mepolizumab group met the two primary end points with significantly more accrued weeks of remission and a higher percentage of patients in remission. However, $47 \%$ of participants did not achieve remission, and it is unclear why certain patients responded better than others. AEs were similar between groups, while serious AEs were slightly higher in the placebo group (26\% versus $18 \%$ ), although some of the events may be attributable to underlying EGPA activity.

RTX has not been studied by controlled trial in EGPA patients, although a few observational studies have reported successful use in both induction and maintenance $[45,46]$. The adjunctive use of PLEX to CYC has been evaluated in an early study, which reported no additional benefit in survival [47].

\section{Pediatric considerations}

Few studies have evaluated the outcomes of pediatric AAV (pAAV) or compared these with adult-onset AAV (aAAV). While some conflicting results exist, it appears that disease severity in pAAV is similar or slightly higher than in aAAV. Rottem et al. prospectively compared 23 pediatric GPA patients with 135 adult-onset patients and found that remission rates, relapse rates, and serious $\mathrm{AE}$ rates were similar [48]. Sacri et al. found that renal impairment occurred in $90 \%$ of pAAV at disease onset, which is more common than reports in aAAV which range from 10 to $20 \%$ at diagnosis to $60-80 \%$ during the disease course [49]. Iudici et al. compared 35 pAAV patients with 151 aAAV patients in a matched case-control study [50]. Both groups received similar induction therapy, most commonly corticosteroids and IV CYC. The authors report that by 5 years, pAAV patients had higher relapse rates, accumulated more damage, and were more likely to remain on corticosteroids and immunosuppressive agents than their adult counterparts. Eleven percent of pAAV and $9 \%$ of aAAV patients died.

To date, there have been no RCTs evaluating treatment regimens in pAAV. The four largest cohort studies assessing contemporary outcomes in pAAV found that the majority of patients received corticosteroids and CYC for remission induction, followed by AZA or MTX for remission maintenance (Table 5). The definitions and rates of remission and relapse varied between studies. Sacri et al. reported disease remission in $92 \%$ of pAAV patients, and a relapse rate of $41 \%$ [51]. In a singlecentre study from Toronto [52], all 20 pAAV followed for a minimum of 6 months achieved remission; the relapse rate was $75 \%$ at a median follow-up of 10 months. Iudici et al. reported inactive disease in all pediatric EGPA and MPA patients and 68\% of GPA patients at the last follow-up visit (median 96 months), however many of these patients continued to be on therapy, including many on corticosteroids [51].

The largest pAAV cohort to date, the ARChiVe (A Registry for Childhood Vasculitis: e-entry) cohort [10], evaluated the outcomes of 105 pAAV patients and found that $42 \%$ achieved remission by 12 months (remission defined as PVAS of 0 on $<0.2 \mathrm{mg} / \mathrm{kg} /$ day corticosteroids or equivalent), and $61 \%$ had inactive disease on higher doses of corticosteroids. All but 3 patients in remission were on maintenance therapy at 12 months, and $48 \%$ had discontinued corticosteroids. Rates of remission were similar for those treated aggressively (CYC and/or RTX, 43\%) versus moderately (MTX, AZA or MMF, 30\%); those treated aggressively had higher baseline PVAS scores. Twenty-four percent of patients experienced minor relapses after achieving inactive disease. The remission rate of $42 \%$ in this study is lower than that reported in the adult studies, which ranges from 53 to $93 \%[14,17,22]$ but direct comparison is complicated by differences in study design, remission definitions, and corticosteroid regimens. Sixtythree percent of the ARChiVe cohort had evidence of damage by 12 months, compared to $87 \%$ reported in grouped adult data [52]. Given that we have speculated greater organ reserve in children compared to adults, this 
Lee et al. Pediatric Rheumatology

(2019) 17:31

Page 9 of 15

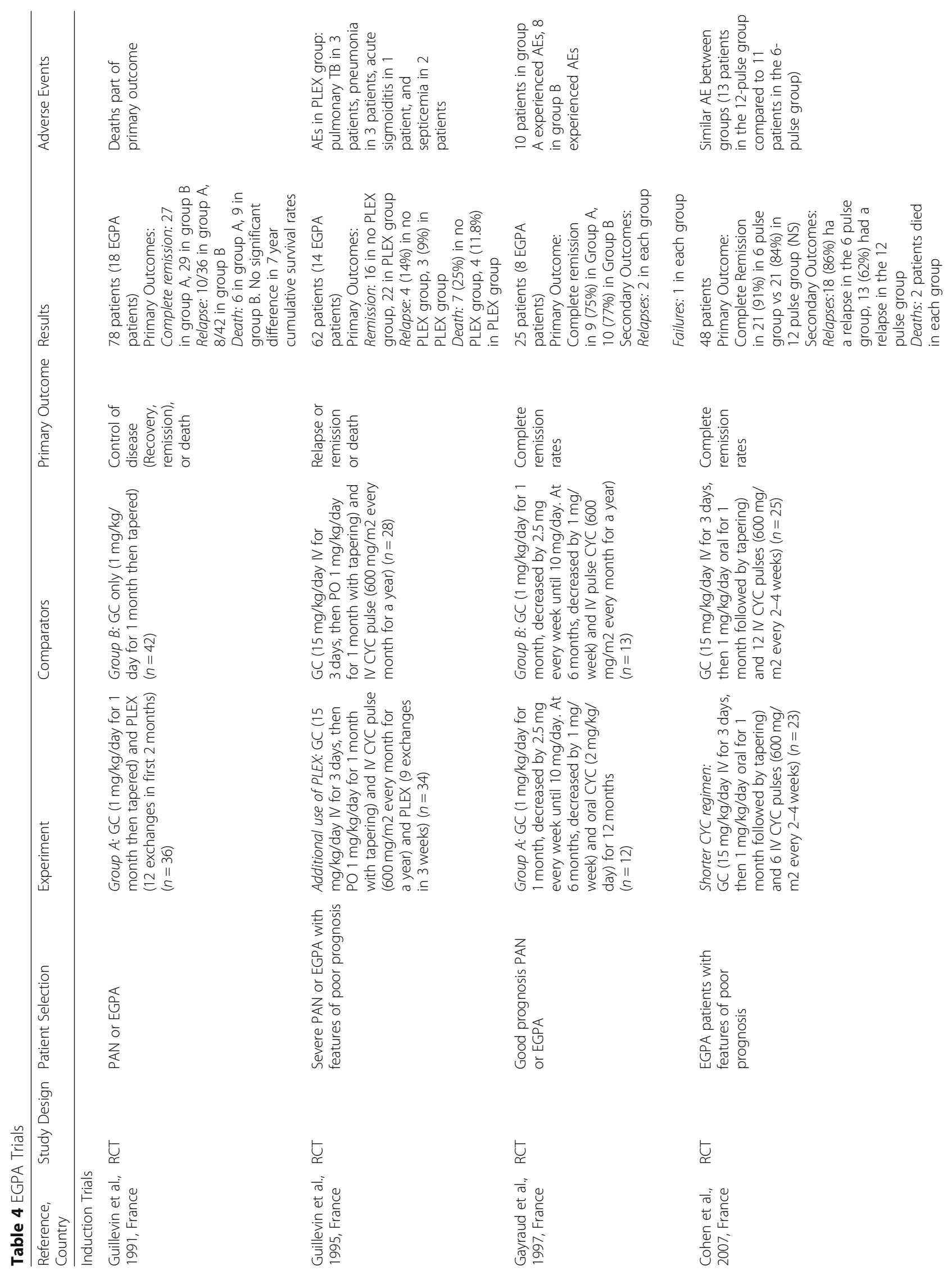




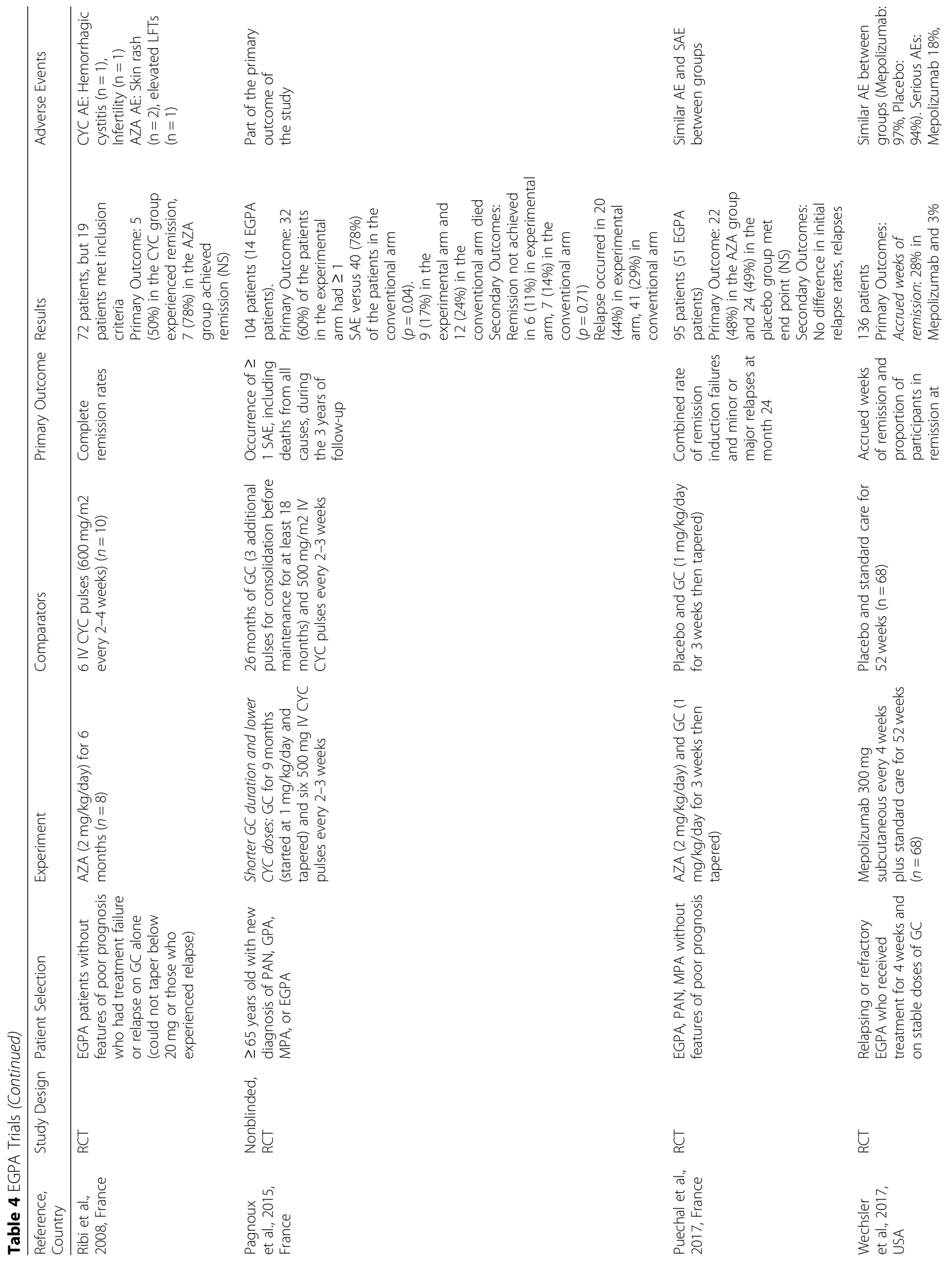


Lee et al. Pediatric Rheumatology

(2019) 17:31

Page 11 of 15

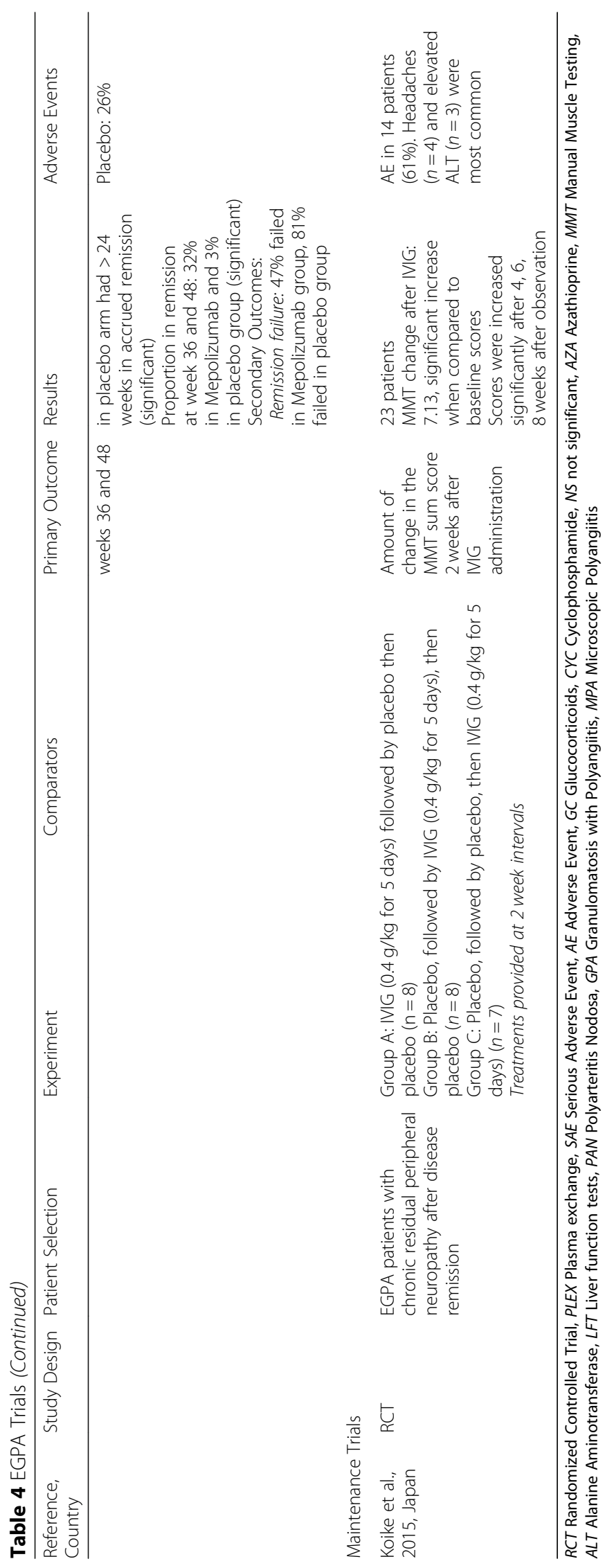




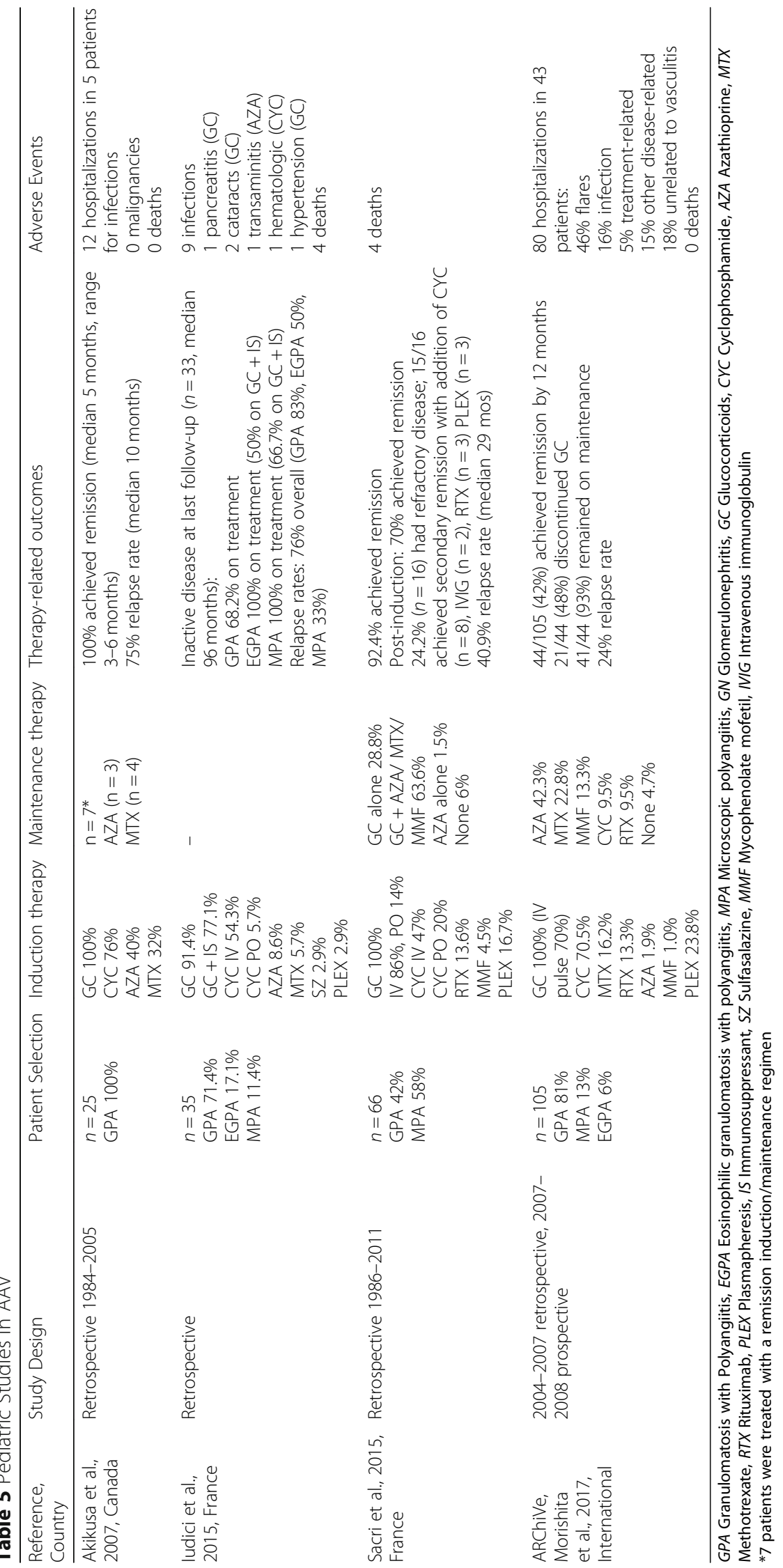


high rate of damage in children only 12 months after diagnosis is concerning and further suggests that children with AAV may have a more severe disease course than their adult counterparts.

The Single Hub and Access point for Pediatric Rheumatology in Europe (SHARE) initiative recently developed consensus-based guidelines for the management of rare pediatric vasculitides. These included recommendations for pAAV (including EGPA) along with Polyarteritis Nodosa and Takayasu Arteritis [53]. Given the paucity of pediatric-specific evidence and highly variable clinical practice amongst various centers, the aims of the recommendations were to define the minimum standard of care for these patients. A large portion of the guidelines is directed at establishing the diagnosis of systemic vasculitis in a pediatric patient.

Adult-derived literature was primarily used to address pAAV treatment recommendations, as the quality of pediatric evidence was poor and largely based on descriptive studies. Induction recommendations for severe disease largely remain similar to adult guidelines, which included corticosteroids and ivCYC as primary agents. The guidelines stress the importance of IV over PO CYC use given the lower cumulative toxicity but similar efficacy. Interestingly, PLEX was defined as a "typical" initial induction agent in severe AAV patients, while adult guidelines generally describe it as an adjunctive agent with insufficient evidence to support its use as a first-line therapy. Furthermore, while adult guidelines strongly support the use of RTX as a first-line remission induction therapy, the same recommendation was not made in the SHARE guidelines, and was considered a second or third line induction agent. Unfortunately, the guidelines do not distinguish severe from limited AAV, and do not provide any specific treatment recommendations for pediatric patients with limited disease, which could potentially lead to confusion or possible over-treatment of a unique subgroup of pAAV patients. Similar agents have been recommended for maintenance therapies.

\section{Future directions}

There are questions that remain unanswered in AAV management. In the realm of induction management, LoVAS (Low-dose Glucocorticoids Plus Rituximab Versus High-dose Glucocorticoids Plus Rituximab for Remission Induction in ANCA-associated Vasculitis) is a trial currently underway to evaluate whether corticosteroid regimens can be used in lower doses when RTX is used as the induction agent [54]. The PEXIVAS study provides some preliminary suggestion that regimens utilizing lower doses of corticosteroids do not impact rates of severe outcomes (such as death or ESRD), but final results are pending [21]. The CLEAR study was a recent trial that demonstrated that Avacopan, a C5 receptor inhibitor, could potentially replace or reduce corticosteroid doses, bringing forward unique therapies with a possible steroid-sparing role [55].

With regards to maintenance, the MAINRITSAN3 is a RCT comparing RTX for 46 months compared to 18 months. The RITAZAREM trial is an ongoing study using higher dose RTX in patients with relapsing disease [56]. TAPIR (The Assessment of Prednisone In Remission Trial) is a trial comparing continuation of low-dose corticosteroid versus stopping corticosteroid entirely in GPA patients during maintenance.

Given that EGPA remains understudied in comparison to the other AAVs, further research is needed to determine the efficacy of conventional immunosuppressants and RTX in EGPA, and the optimal patient candidates and dosing regimen for mepolizumab. ANCA-negative patients, excluded by most trials to date, also remain understudied, constituting another future research priority.

Evidence-based guidelines for pAAV management remain sparse. The EULAR/EUVAS guidelines do not comment on the pediatric population. The CanVasc recommendations make 4 pediatric-specific statements, primarily suggesting that pediatric patients should be treated according to adult guidelines [35]. As previously mentioned, the SHARE guidelines for the management of pAAV have been developed in order to set the minimum standard of care when it comes to systemic vasculitis treatment [53]. The majority of treatment recommendations however, were based on low-quality pediatric evidence, expert opinion, or extrapolated from adult studies. While these recent guidelines address the pediatric rheumatology community's desire and need for more pediatric specific recommendations [57], evaluating its uptake and usefulness is warranted. Additional multi-center studies in pAAV are required to address questions around efficacy and toxicity of existing therapies in the pediatric setting, so that pediatric guidelines may incorporate higher quality evidence.

\section{Conclusion}

Significant progress has been made in our understanding of the management and outcomes of AAV over the last two decades. Future studies should be directed towards addressing the remaining unanswered questions, which include determining the optimal duration and regimen of AAV induction and maintenance therapy, improving our understanding of EGPA management, and developing evidence for pAAV to better inform pediatric-specific treatment guidelines.

\section{Abbreviations}

aAAV: Adult-onset ANCA-Associated Vasculitis; AAV: ANCA-associated

Vasculitis; AEs: Adverse Events; ANCA: Anti-neutrophil cytoplasmic antibody; AZA: Azathioprine; BVAS: Birmingham Vasculitis Activity Score;

CanVasc: Canadian Vasculitis research network; CARRA: Childhood Arthritis \& 
Rheumatology Research Alliance; CYC: Cyclophosphamide; EGPA: Eosinophilic Granulomatosis with Polyangiitis; ESRD: End stage Renal Disease; EULAR: European League Against Rheumatism; GPA: Granulomatosis with Polyangiitis; ivCYC: Intravenous Cyclophosphamide;

MMF: Mycophenolate Mofetil; MPA: Microscopic Polyangiitis;

MTX: Methotrexate; pAAV: Pediatric ANCA-Associated Vasculitis; PLEX: Plasma Exchange; poCYC: Oral Cyclophosphamide; poMTX: Oral Methotrexate; PRES: Pediatric Rheumatology European Society; PVAS: Pediatric Vasculitis Activity Score; RCT: Randomized Controlled Trial; RTX: Rituximab; SHARE: Single Hub and Access point for Pediatric Rheumatology in Europe; VDI: Vasculitis Damage Index

\section{Acknowledgements}

RSMY is supported by the Hak-Ming and Deborah Chiu Chair in Paediatric Translational Research at the Hospital for Sick Children, University of Toronto.

\section{Authors' contributions}

$J J Y L, A A, G P K C$, EL, WS conceptualized the design of the review, completed the literature search, selected the papers for inclusion, created figures and tables, contributed equally to the draft of the manuscript, and reviewed the manuscript. RSMY, JA, and RML were consulted for guidance on the appropriateness of the article. RML supervised the conceptual design of the review. RSMY, JA, and RML reviewed and critically revised the manuscript. All authors approved the final manuscript as submitted and agree to be accountable for all aspects of the work.

\section{Authors' information}

Not applicable.

\section{Funding}

This project did not receive any direct funding.

\section{Availability of data and materials}

Not applicable. Published data that support the findings of this study are included in the article as part of the review.

\section{Ethics approval and consent to participate}

Not applicable.

\section{Consent for publication}

Not applicable.

\section{Competing interests}

The authors declare that they have no competing interests.

\section{Author details}

'Department of Pediatrics, Division of Rheumatology, Hospital for Sick Children and University of Toronto, 555 University Avenue, Toronto, ON M5G 1X8, Canada. ${ }^{2}$ Department of Pediatrics and Adolescent Medicine, Alice Ho Miu Ling Nethersole Hospital, HKSAR, Tai Po, Hong Kong. ${ }^{3}$ Department of Pediatrics, Faculty of Medicine, Chiang Mai University, Chiang Mai, Thailand. ${ }^{4}$ Department of Rheumatology, The Royal Children's Hospital, Melbourne, Australia.

\section{Received: 17 May 2019 Accepted: 20 June 2019}

Published online: 26 June 2019

\section{References}

1. Phillip R, Luqmani R. Mortality in systemic vasculitis: a systematic review. Clin Exp Rheumatol. 2008;26(5 Suppl 51):S94-104.

2. Lane SE, Watts RA, Shepstone L, Scott DG. Primary systemic vasculitis: clinical features and mortality. QJM. 2005;98(2):97-111.

3. Kallenberg CG, Tervaert JW, Stegeman CA. Criteria for disease activity in Wegener's granulomatosis: a requirement for longitudinal clinical studies. APMIS Suppl. 1990;19:37-9.

4. de Groot K, Gross WL, Herlyn K, Reinhold-Keller E. Development and validation of a disease extent index for Wegener's granulomatosis. Clin Nephrol. 2001;55(1):31-8

5. Whiting-O'Keefe QE, Stone JH, Hellmann DB. Validity of a vasculitis activity index for systemic necrotizing vasculitis. Arthritis Rheum. 1999; 42(11):2365-71.
6. Luqmani RA, Bacon PA, Moots RJ, Janssen BA, Pall A, Emery P, et al. Birmingham Vasculitis activity score (BVAS) in systemic necrotizing vasculitis. QJM. 1994;87(11):671-8.

7. Mukhtyar C, Lee R, Brown D, Carruthers D, Dasgupta B, Dubey S, et al. Modification and validation of the Birmingham Vasculitis activity score (version 3). Ann Rheum Dis. 2009;68(12):1827.

8. Stone JH, Hoffman GS, Merkel PA, Min Yl, Uhlfelder ML, Hellmann DB, et al. A disease-specific activity index for Wegener's granulomatosis: modification of the Birmingham Vasculitis activity score. Arthritis and Rheum. 2001;44(4): 912-20.

9. Dolezalova P, Price-Kuehne FE, Ozen S, Benseler SM, Cabral DA, Anton J, et al. Disease activity assessment in childhood vasculitis: development and preliminary validation of the Paediatric Vasculitis activity score (PVAS). Ann Rheum Dis. 2013: 72(10):1628-33.

10. Morishita KA, Moorthy LN, Lubieniecka JM, Twilt M, Yeung RSM, Toth MB, et al. Early outcomes in children with Antineutrophil cytoplasmic antibodyassociated Vasculitis. Arthritis Rheumatol. 2017:69(7):1470-9.

11. Exley AR, Bacon PA, Luqmani RA, Kitas GD, Gordon C, Savage CO, et al. Development and initial validation of the Vasculitis damage index for the standardized clinical assessment of damage in the systemic vasculitides. Arthritis Rheum. 1997:40(2):371-80.

12. Hellmich B, Flossmann O, Gross WL, Bacon P, Cohen-Tervaert JW, Guillevin $L$, et al. EULAR recommendations for conducting clinical studies and/or clinical trials in systemic vasculitis: focus on anti-neutrophil cytoplasm antibody-associated vasculitis. Ann Rheum Dis. 2007;66(5):605-17.

13. McGeoch L, Twilt M, Famorca L, Bakowsky V, Barra L, Benseler SM, et al. CanVasc recommendations for the Management of Antineutrophil Cytoplasm Antibody-associated Vasculitides. J Rheumatol. 2016;43(1):97.

14. de Groot K, Harper L, Jayne DR, Flores Suarez LF, Gregorini G, Gross WL, et al. Pulse versus daily oral cyclophosphamide for induction of remission in antineutrophil cytoplasmic antibody-associated vasculitis: a randomized trial. Ann Intern Med. 2009; 150(10):670-80.

15. Jones RB, Cohen Tervaert JW, Hauser T, Luqmani R, Morgan MD, Peh CA, et al. Rituximab versus cyclophosphamide in ANCA-associated renal Vasculitis. N Engl J Med. 2010;363(3):211-20.

16. Jones RB, Furuta S, Tervaert JW, Hauser T, Luqmani R, Morgan MD, et al. Rituximab versus cyclophosphamide in ANCA-associated renal vasculitis: 2year results of a randomised trial. Ann Rheum Dis. 2015;74(6):1178-82.

17. Stone JH, Merkel PA, Spiera R, Seo P, Langford CA, Hoffman GS, et al. Rituximab versus cyclophosphamide for ANCA-associated Vasculitis. N Engl Med. 2010;363(3):221-32

18. Specks U, Merkel PA, Seo P, Spiera R, Langford CA, Hoffman GS, et al. Efficacy of remission-induction regimens for ANCA-associated vasculitis. N Engl J Med. 2013;369(5):417-27.

19. Walsh M, Merkel PA, Peh CA, Szpirt W, Guillevin L, Pusey CD, et al. Plasma exchange and glucocorticoid dosing in the treatment of anti-neutrophil cytoplasm antibody associated vasculitis (PEXIVAS): protocol for a randomized controlled trial. Trials. 2013;14:73.

20. Walsh M, Casian A, Flossmann O, Westman K, Hoglund P, Pusey C, et al. Long-term follow-up of patients with severe ANCA-associated vasculitis comparing plasma exchange to intravenous methylprednisolone treatment is unclear. Kidney Int. 2013:84(2):397-402.

21. Walsh M, Merkel PA, Jayne D. The effects of plasma exchange and reduceddose glucocorticoids during remission-induction for treatment of severe ANCA-associated Vasculitis [abstract]. Arthritis Rheumatol. 2018;70(suppl 10). https://acrabstracts.org/abstract/the-effects-of-plasma-exchange-andreduced-dose-glucocorticoids-during-remission-induction-for-treatment-ofsevere-anca-associated-vasculitis/. Accessed 24 June 2019.

22. De Groot K, Rasmussen N, Bacon PA, Tervaert JW, Feighery C, Gregorini G, et al. Randomized trial of cyclophosphamide versus methotrexate for induction of remission in early systemic antineutrophil cytoplasmic antibody-associated vasculitis. Arthritis Rheum. 2005;52(8):2461-9.

23. Faurschou M, Westman $K$, Rasmussen N, de Groot K, Flossmann O, Hoglund $P$, et al. Brief report: long-term outcome of a randomized clinical trial comparing methotrexate to cyclophosphamide for remission induction in early systemic antineutrophil cytoplasmic antibody-associated vasculitis. Arthritis Rheum. 2012; 64(10):3472-7.

24. Jones RB, Hiemstra TF, Ballarin J, Blockmans DE, Brogan P, Bruchfeld A, et al. Mycophenolate mofetil versus cyclophosphamide for remission induction in ANCA-associated vasculitis: a randomised, non-inferiority trial. Ann Rheum Dis. 2019;78(3):399-405. 
25. Jayne D, Rasmussen N, Andrassy K, Bacon P, Tervaert JW, Dadoniene J, et al. A randomized trial of maintenance therapy for vasculitis associated with antineutrophil cytoplasmic autoantibodies. N Engl J Med. 2003;349(1):36-44.

26. Walsh M, Faurschou M, Berden A, Flossmann O, Bajema I, Hoglund P, et al. Long-term follow-up of cyclophosphamide compared with azathioprine for initial maintenance therapy in ANCA-associated vasculitis. Clin J Am Soc Nephrol. 2014;9(9):1571-6.

27. Karras A, Pagnoux C, Haubitz M, Groot K, Puechal X, Tervaert JWC, et al. Randomised controlled trial of prolonged treatment in the remission phase of ANCA-associated vasculitis. Ann Rheum Dis. 2017;76(10):1662-8.

28. de Joode AAE, Stegeman CA, Sanders J-SF, DeSevaux RG, Broekroelofs J, Voskuyl $A E$, et al. Extended versus standard azathioprine maintenance therapy in newly diagnosed proteinase-3 anti-neutrophil cytoplasmic antibody-associated vasculitis patients who remain cytoplasmic antineutrophil cytoplasmic antibody-positive after induction of remission: a randomized clinical trial. Nephrol Dial Transplant. 2016;31(9):1453-9.

29. Pagnoux C, Mahr A, Hamidou MA, Boffa JJ, Ruivard M, Ducroix JP, et al. Azathioprine or methotrexate maintenance for ANCA-associated vasculitis. N Engl J Med. 2008;359(26):2790-803.

30. Puechal X, Pagnoux C, Perrodeau E, Hamidou M, Boffa JJ, Kyndt X, et al. Long-term outcomes among participants in the WEGENT trial of remissionmaintenance therapy for granulomatosis with Polyangiitis (Wegener's) or microscopic Polyangiitis. Arthritis Rheumatol. 2016;68(3):690-701.

31. Hiemstra TF, Walsh M, Mahr A, Savage CO, de Groot K, Harper L, et al. Mycophenolate mofetil vs azathioprine for remission maintenance in antineutrophil cytoplasmic antibody-associated vasculitis: a randomized controlled trial. Jama. 2010;304(21):2381-8.

32. Metzler C, Miehle N, Manger K, Iking-Konert C, de Groot K, Hellmich B, et al. Elevated relapse rate under oral methotrexate versus leflunomide for maintenance of remission in Wegener's granulomatosis. Rheumatology (Oxford). 2007;46(7):1087-91.

33. Guillevin L, Pagnoux C, Karras A, Khouatra C, Aumaitre O, Cohen P, et al. Rituximab versus azathioprine for maintenance in ANCA-associated vasculitis. N Engl J Med. 2014;371(19):1771-80.

34. Terrier B, Pagnoux C, Perrodeau E, Karras A, Khouatra C, Aumaitre O, et al. Long-term efficacy of remission-maintenance regimens for ANCA-associated vasculitides. Ann Rheum Dis. 2018;77(8):1150-6.

35. Charles $P$, Terrier B, Perrodeau E, Cohen P, Faguer S, Huart A, et al. Comparison of individually tailored versus fixed-schedule rituximab regimen to maintain ANCA-associated vasculitis remission: results of a multicentre, randomised controlled, phase III trial (MAINRITSAN2). Ann Rheum Dis. 2018; 77(8):1143-9.

36. Jayne D, Blockmans D, Luqmani R, Moiseev S, Ji B, Green Y, et al. Efficacy and safety of Belimumab and azathioprine for maintenance of remission in Antineutrophil cytoplasmic antibody-associated Vasculitis: a randomized controlled study. Arthritis Rheumatol. 2019;71(6):952-63.

37. Fauci AS, Katz P, Haynes BF, Wolff SM. Cyclophosphamide therapy of severe systemic necrotizing Vasculitis. N Engl J Med. 1979;301(5):235-8.

38. Cohen P, Pagnoux C, Mahr A, Arene JP, Mouthon L, Le Guern V, et al. Churg-Strauss syndrome with poor-prognosis factors: a prospective multicenter trial comparing glucocorticoids and six or twelve cyclophosphamide pulses in forty-eight patients. Arthritis Rheum. 2007; 57(4):686-93.

39. Guillevin L, Lhote F, Cohen P, Jarrousse B, Lortholary O, Genereau T, et al. Corticosteroids plus pulse cyclophosphamide and plasma exchanges versus corticosteroids plus pulse cyclophosphamide alone in the treatment of polyarteritis nodosa and Churg-Strauss syndrome patients with factors predicting poor prognosis. A prospective, randomized trial in sixty-two patients. Arthritis Rheum. 1995;38(11):1638-45.

40. Gayraud M, Guillevin L, Cohen P, Lhote F, Cacoub P, Deblois P, et al. Treatment of good-prognosis polyarteritis nodosa and Churg-Strauss syndrome: comparison of steroids and oral or pulse cyclophosphamide in 25 patients. Br J Rheumatol. 1997;36(12):1290-7.

41. Groh M, Pagnoux C, Baldini C, Bel E, Bottero P, Cottin V, et al. Eosinophilic granulomatosis with polyangiitis (Churg\&\#x2013;Strauss) (EGPA) Consensus Task Force recommendations for evaluation and management. Eur J Intern Med. 2015;26(7):545-53.

42. Ribi C, Cohen P, Pagnoux C, Mahr A, Arene JP, Lauque D, et al. Treatment of Churg-Strauss syndrome without poor-prognosis factors: a multicenter, prospective, randomized, open-label study of seventy-two patients. Arthritis Rheum. 2008;58(2):586-94.
43. Puechal X, Pagnoux C, Baron G, Quemeneur T, Neel A, Agard C, et al. Adding azathioprine to remission-induction glucocorticoids for eosinophilic granulomatosis with Polyangiitis (Churg-Strauss), microscopic Polyangiitis, or Polyarteritis Nodosa without poor prognosis factors: a randomized, controlled trial. Arthritis Rheumatol. 2017;69(11):2175-86.

44. Wechsler ME, Akuthota P, Jayne D, Khoury P, Klion A, Langford CA, et al. Mepolizumab or placebo for eosinophilic granulomatosis with Polyangiitis. N Engl J Med. 2017;376(20):1921-32.

45. Cartin-Ceba R, Keogh KA, Specks U, Sethi S, Fervenza FC. Rituximab for the treatment of Churg-Strauss syndrome with renal involvement. Nephrol Dial Transplant. 2011;26(9):2865-71.

46. Charles P, Neel A, Tieulie N, Hot A, Pugnet G, Decaux O, et al. Rituximab for induction and maintenance treatment of ANCA-associated vasculitides: multicentre retrospective study on 80 patients. Rheumatology (Oxford). 2014; 53(3):532-9.

47. Guillevin L, Fain O, Lhote F, Jarrousse B, Le Thi Huong D, Bussel A, et al. Lack of superiority of steroids plus plasma exchange to steroids alone in the treatment of polyarteritis nodosa and Churg-Strauss syndrome. A prospective, randomized trial in 78 patients. Arthritis Rheum. 1992;35(2):208-15.

48. Rottem M, Fauci AS, Hallahan CW, Kerr GS, Lebovics R, Leavitt RY, et al. Wegener granulomatosis in children and adolescents: clinical presentation and outcome. J Pediatr. 1993;122(1):26-31.

49. Frosch $M$, Foell D. Wegener granulomatosis in childhood and adolescence. Eur J Pediatr. 2004;163(8):425-34.

50. Iudici M, Pagnoux C, Quartier P, Buchler M, Cevallos R, Cohen P, et al. Childhood- versus adult-onset ANCA-associated vasculitides: a nested, matched case-control study from the French Vasculitis study group registry. Autoimmun Rev. 2018;17(2):108-14.

51. ludici M, Puechal X, Pagnoux C, Quartier P, Agard C, Aouba A, et al. Brief report: childhood-onset systemic necrotizing Vasculitides: long-term data from the French Vasculitis study group registry. Arthritis Rheumatol. 2015;67(7):1959-65.

52. Robson J, Doll H, Suppiah R, Flossmann O, Harper L, Hoglund P, et al. Damage in the anca-associated vasculitides: long-term data from the European vasculitis study group (EUVAS) therapeutic trials. Ann Rheum Dis. 2015;74(1):177-84.

53. de Graeff N, Groot N, Brogan P, Ozen S, Avcin T, Bader-Meunier B, et al. European consensus-based recommendations for the diagnosis and treatment of rare paediatric vasculitides - the SHARE initiative. Oxford, England: Rheumatology; 2018.

54. Furuta S, Sugiyama T, Umibe T, Kaneko Y, Amano K, Kurasawa K, et al. Low-dose glucocorticoids plus rituximab versus high-dose glucocorticoids plus rituximab for remission induction in ANCA-associated vasculitis (LOVAS): protocol for a multicentre, open-label, randomised controlled trial. BMJ Open. 2017;7(12):e018748.

55. Jayne DRW, Bruchfeld AN, Harper L, Schaier M, Venning MC, Hamilton P, et al. Randomized trial of C5a receptor inhibitor Avacopan in ANCAassociated Vasculitis. J Am Soc Nephrol. 2017;28(9):2756-67.

56. Gopaluni S, Smith RM, Lewin M, McAlear CA, Mynard K, Jones RB, et al. Rituximab versus azathioprine as therapy for maintenance of remission for anti-neutrophil cytoplasm antibody-associated vasculitis (RITAZAREM): study protocol for a randomized controlled trial. Trials. 2017;18(1):112.

57. Westwell-Roper C, Lubieniecka JM, Brown KL, Morishita KA, Mammen C, Wagner-Weiner $L$, et al. Clinical practice variation and need for pediatricspecific treatment guidelines among rheumatologists caring for children with ANCA-associated vasculitis: an international clinician survey. Pediatr Rheumatol Online J. 2017;15(1):61.

\section{Publisher's Note}

Springer Nature remains neutral with regard to jurisdictional claims in published maps and institutional affiliations. 